\title{
Orbital tomography for highly symmetric adsorbate systems
}

\author{
B. Stadtmüller ${ }^{1,2}$, M. Willenbockel ${ }^{1,2}$, E. M. Reinisch ${ }^{3}$, T. $\operatorname{Ules}^{3}$, F. C. Bocquet $^{1,2}$, S. Soubatch $^{1,2}$,

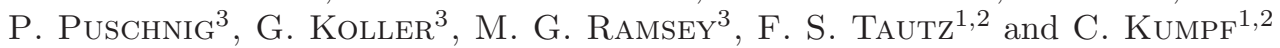 \\ 1 Peter Grünberg Institut (PGI-3), Forschungszentrum Jülich - 52425 Jülich, Germany \\ 2 Jülich Aachen Research Alliance (JARA)-Fundamentals of Future Information Technology - 52425 Jülich, Germany \\ 3 Institut für Physik, Karl-Franzens-Universität Graz-8010 Graz, Austria
}

\begin{abstract}
PACS 68.43. $-\mathrm{h}$ - Chemisorption/physisorption: adsorbates on surfaces
PACS 81.05. Fb - Organic semiconductors in materials science

PACS 79.60.Dp - Photoemission from adsorbed layers
\end{abstract}

\begin{abstract}
Orbital tomography is a new and very powerful tool to analyze the angular distribution of a photoemission spectroscopy experiment. It was successfully used for organic adsorbate systems to identify (and consequently deconvolute) the contributions of specific molecular orbitals to the photoemission data. However, the technique was so far limited to surfaces with low symmetry like fcc (110) oriented surfaces, owing to the small number of rotational domains that occur on such surfaces. In this letter we overcome this limitation and present an orbital tomography study of a 3,4,9,10-perylene-tetra-carboxylic-dianhydride (PTCDA) monolayer film adsorbed on Ag (111). Although this system exhibits up to twelve differently oriented molecules, the angular resolved photoemission data still allow a meaningful analysis of the different local density of states and reveal different electronic structures for symmetrically inequivalent molecules. We also discuss the precision of the orbital tomography technique in terms of counting statistics and the linear regression fitting algorithm. Our results demonstrate that the orbital tomography method is not limited to low-symmetry surfaces, a finding which makes a broad field of complex adsorbate systems accessible to this powerful technique.
\end{abstract}

Introduction. - The alignment of the electronic levels at interfaces between different materials plays a crucial role for the functionality of (organic) electronic devices. In particular, the states close to the Fermi level are important, because they dominate the charge transport through the interface. Furthermore, the lowest unoccupied and highest occupied molecular orbitals (LUMO and HOMO) play an important role in the interaction of molecules across the interface since they, e.g., determine the bonding mechanisms of molecules on surfaces. A direct access to the occupied frontier orbitals can be provided by ultra violet photoelectron spectroscopy (UPS) which allows a very precise determination of the energetic positions of the occupied molecular orbitals as well as the substrate states (see, e.g., [1] and references therein). This makes UPS one of the most popular experimental techniques for evaluating the electronic properties of interfaces in general. However, the assignment of the features in the photoemission spectra to molecular states is not easy, in conventional (angle integrated) UPS it is often even impossible without additional knowledge about the system. Charge redistribution at and across the interface can lead to both population and depopulation of the individual molecular levels, even simultaneously within the same molecule [2-6]. In addition, small differences in the electronic structure of the individual molecules, e.g., caused by a different orientation on the substrate, can usually not be resolved due to relatively broad spectral features of the molecular states.

To some extent these limitations of UPS can be overcome by measuring the angular distribution of the photoemission yield, i.e., by angular resolved photoemission spectroscopy (ARPES). The momentum distribution of the photoemission intensity from a specific molecular orbital can be understood in terms of the square of the Fourier transform of the molecular wave function by approximating the final state as a plane wave. This opens the possibility to identify the molecular orbital from its ARPES signature [7]. Recently this idea has been improved by establishing the "orbital tomography" technique which allows to deconvolute the contributions of specific molecu- 
lar orbitals to experimental ARPES data [8]. The same technique can also be used to separate the spectral contributions of different (or differently oriented) molecules in the spectra, as long as their momentum distribution is not identical [9]. Therefore, this new technique holds great potential for investigating the arrangements of molecules in the unit cell, their orientations with respect to the substrate, and consequently allows to evaluate the complex interplay between intermolecular and molecule-substrate interactions [8].

To the best of our knowledge the orbital tomography approach has only been applied to (110)-oriented surfaces of face centered cubic crystals so far [7-11]. This is clearly due to the fact that this surface shows only a low $p 2 \mathrm{~mm}$ symmetry which allows only few different molecular orientations, since most molecules (especially elongated molecules) tend to align along high symmetry directions of the substrate surface. For 3,4,9,10-perylene-tetracarboxylic-dianhydride (PTCDA) on $\mathrm{Ag}(110)$, for example, only one molecular orientation occurs. Hence, in this case the angular distribution of the photoelectron yield is comparable to the emission of one single molecule. In that sense low-symmetric surfaces represent the simplest cases which are expected to give unambiguous results. Adsorbate systems on higher symmetric surfaces, however, were so far considered to be too difficult to be analyzed by orbital tomography.

In this work we attack this perceived limitation of the technique by investigating a very popular adsorbate system on the $\operatorname{Ag}(111)$ surface having $p 3 m 1$ symmetry: The monolayer structure of PTCDA on $\mathrm{Ag}(111)$ [12-18]. The unit cell contains two inequivalent molecules with distinct azimuthal orientations [19]. Due to three rotational domains, each having one additional mirror domain, we hence have to deal with 12 molecules with different orientations on the surface. This situation makes PTCDA/Ag(111) the ideal challenge for the orbital tomography technique. Moreover, the local density of states of the two inequivalent molecules has been studied by scanning tunneling spectroscopy (STS) [19] which can be used as reference data. The molecular orbitals which were visible in the occupied regime could be assigned to the HOMO and the former LUMO level, the latter becoming partially filled by the interaction with the substrate and therefore cut by the Fermi level.

Experimental. - The ARPES experiments were performed at beamline U125/2-SGM of the BESSY II storage ring, Berlin. The samples have been prepared under UHV conditions (base pressure $<5 \times 10^{-10}$ mbar) by depositing the organic material from dedicated evaporation cells onto a clean $\mathrm{Ag}$ (111) surface. After deposition of a multilayer film of PTCDA the sample was annealed at $280^{\circ} \mathrm{C}$. This procedure causes desorption of the 2nd and higher molecular layers and leaves behind a nearly perfectly ordered monolayer structure of PTCDA/Ag(111) [13]. The success of the preparation process was verified by LEED.
The photoelectron yield was recorded with a toroidal electron analyzer having a polar acceptance angle of $\pm 80^{\circ}$ and an energy dispersion range of $1.0 \mathrm{eV}$ at a pass energy of $10 \mathrm{eV}$. At a photon energy of the synchrotron light of $h \nu=35 \mathrm{eV}$ the energy resolution of the setup is better than $150 \mathrm{meV}$. The azimuthal angular distribution was accessed by rotating the sample around its surface normal in steps of $1^{\circ}$ in a range of at least $130^{\circ}$. Transforming the data from polar and azimuthal emission angles to $k_{x}$ and $k_{y}$ coordinates and considering the threefold symmetry of the silver bulk crystal yields a three dimensional ARPES data cube $I\left(k_{x}, k_{y}, E_{b}\right)$ (cf. supplementary material). Such data cubes have been recorded for each resonance found in angle integrated UPS spectra measured beforehand [13]. In order to account for the substrate contribution, ARPES data of the clean substrate were recorded in the same energy regions. From the experimental data cubes momentum space maps at constant binding energy can be extracted for comparison with theoretical maps calculated from specific molecular orbitals. This allows to assign the recorded spectral features to molecular orbitals, as described in the following.

Theoretical constant binding energy maps. - It has been shown that the ARPES intensity, that is emitted by one individual molecular orbital, can be calculated from the wave function of the orbital. An intensity map of such ARPES data can be obtained from a hemispherical cut through the three dimensional Fourier transform of the molecular orbital wave function at a radius $|k|$ which is determined by the kinetic energy of the electrons in the final state [8]. Panels a and b of Fig. 1 show such ARPES maps for the HOMO and LUMO, respectively, for one individual PTCDA molecule which is oriented with its long axis in horizontal direction in the Figure. However, for computing the ARPES intensity for a PTCDA/Ag(111) monolayer film, the in-plane orientation of the PTCDA molecules in the layer need to be considered. These orientations are adopted from high resolution STM data [19]. There are two molecules in an almost rectangular unit cell. One is almost precisely aligned with its long axis along the silver atom rows, i.e., along the [101] high symmetry direction of the substrate. We label this molecule "A" in the following. The second molecule "B" is rotated $77^{\circ}$ with respect to molecule $\mathrm{A}$. These orientations and the six-fold symmetry of the uppermost layer of the $\mathrm{Ag}(111)$ are employed to generate the calculated constant binding energy (CBE) maps which we present in Fig. 1(c-e). In panel c and $\mathrm{d}$ the molecular orientation is $0^{\circ}$ with respect to the [101] (or equivalent) substrate directions as it is the case for molecule A, in Fig. 1e and $\mathrm{f}$ it is $77^{\circ}$, corresponding to the orientation of molecule B. Note that the orientations of the molecules in some domains coincide in the case of molecule A since it lies parallel to the mirror lines of the structure. For this reason the LUMO emission pattern shown in Fig. 1d looks as if only three molecular orientations were averaged. In case of molecule B the misalign- 


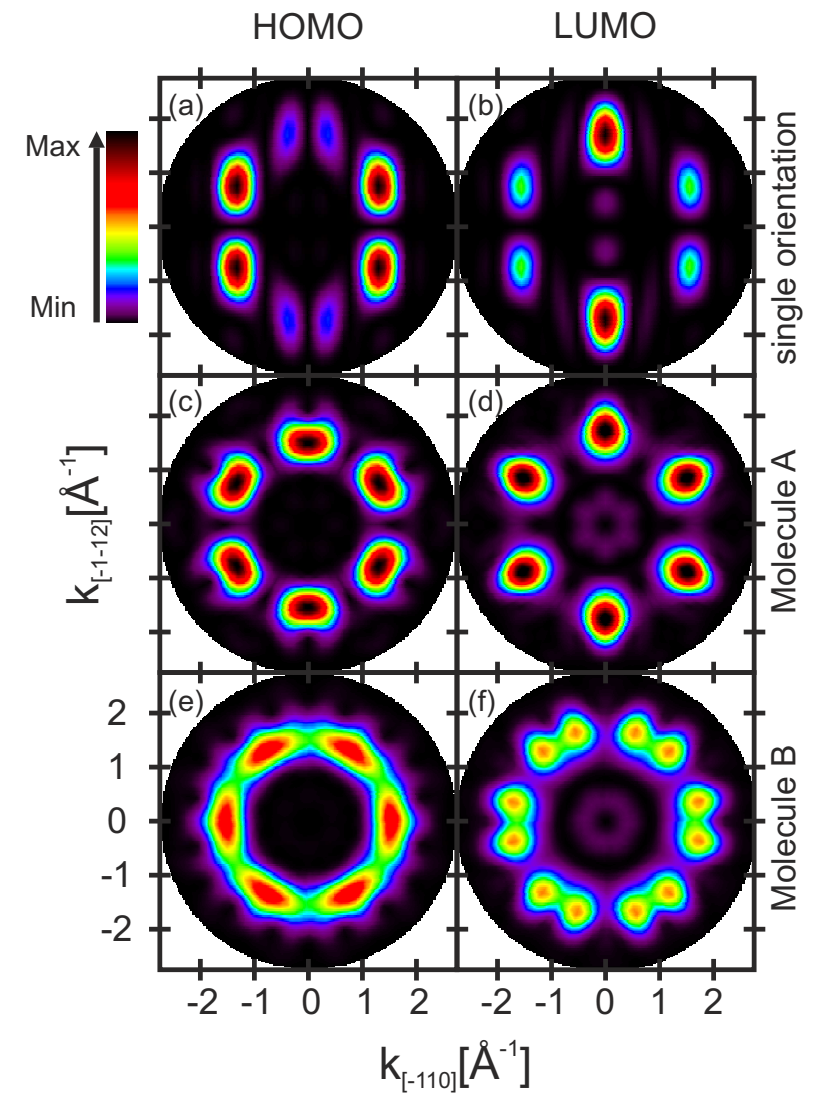

Fig. 1: Constant binding energy (CBE) maps for the HOMO and LUMO level of PTCDA molecules calculated by density functional theory. In (a) and (b) CBE maps for a free molecule with the long axes aligned horizontally are shown. In the other panels the $\mathrm{p} 3 \mathrm{~m} 1$ symmetry of the substrate surface is considered, which causes a superposition of six different molecular orientations. In (c) and (d) this is performed for molecule A (aligned along [101]), in (e) and (f) for molecule B ( $77^{\circ}$ with respect to $[\overline{101}])$.

ment of the molecule with respect to the high symmetry direction becomes clearly visible. It leads to a splitting of the emission maxima by $\pm 17^{\circ}$ as can be seen in panel (f) for the LUMO states, and in case of the HOMO emission even smears out the intensity to an almost continuous ring (Fig. 1e).

Orbital Tomography: Deconvoluting the data. -

In Fig. 2a and b calculated CBE maps for the overall emission from the PTCDA monolayer structure are shown. The maps correspond to the sum of the maps for molecules $\mathrm{A}$ and B, i.e., Fig. 1c+e for the HOMO and $\mathrm{d}+\mathrm{f}$ for the LUMO. Note that this calculation requires the HOMOs (and also the LUMOs) of both molecules to be located at the same binding energy. Although this is not strictly fulfilled in our case (see below), a qualitative evaluation is still possible. Later this energy discrepancy will be taken into account. Note that both CBE maps are dominated by the pattern of molecule A, owing to the higher maximum intensity in these maps caused by the coincidence of

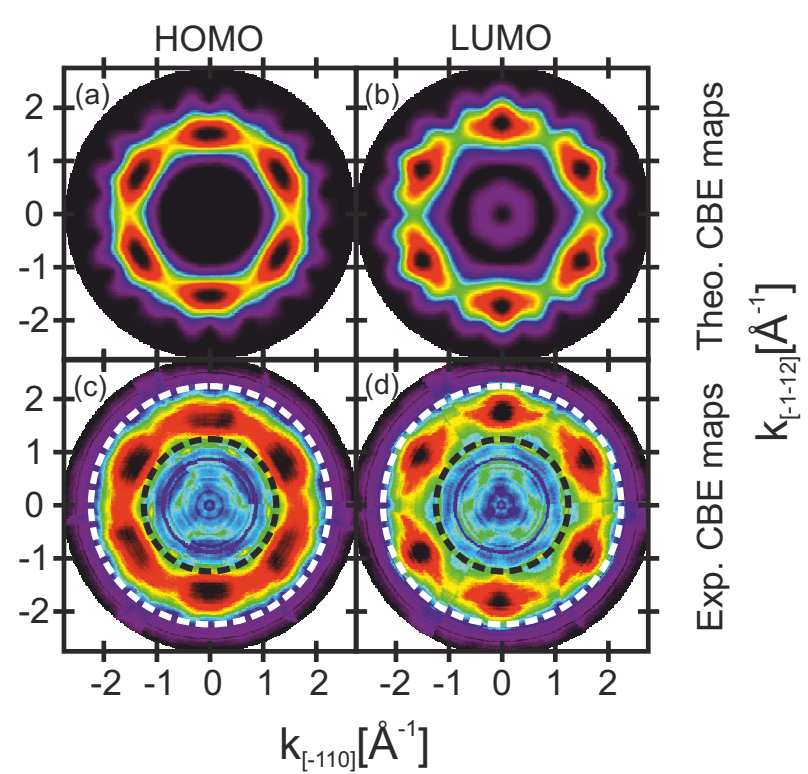

Fig. 2: Calculated (a, b) and measured (c, d) CBE maps of the HOMO and LUMO level, respectively, for the monolayer structure of PTCDA/Ag(111). Calculated maps consider surface symmetry and different molecular orientations (compare with Fig. 1). Experimental maps were recorded for $E_{b}=1.6 \mathrm{eV}$ (c) and $E_{b}=0.3 \mathrm{eV}(\mathrm{d})$.

several peak positions (see above).

In Fig. 2c and d corresponding experimental data are shown. The CBEs represent cuts through the experimental data cubes at binding energies of $E_{b}=1.6 \mathrm{eV}$ and $0.3 \mathrm{eV}$ for HOMO and LUMO, respectively. They match the predicted maps for the HOMO (panel a) and LUMO (b) very well. Note that this also confirms that the peak at the Fermi edge, which is frequently found by various spectroscopic methods [13,19], in fact is the (former) LUMO state which is shifted towards the Fermi energy and (partly) filled due to the interaction of the PTCDA molecule with the surface; i.e., the widely accepted assignment of molecular orbitals and spectral resonances is proven to be correct. This analysis already allows to conclude that the ARPES yield for a molecular adsorbate system exhibiting six rotational and mirror domains can be qualitative understood by the concept of the plane wave approximation for the PES final state.

For a more quantitative analysis of the ARPES data of Fig. 2 c-d we now use a tomographic approach to deconvolute the measured ARPES intensity and extract the contributions of the orbitals of the two individual molecules to the density of states. To this end we employ a procedure similar to that introduced by Puschnig et al. [8] and use the function

$$
\begin{aligned}
F\left(k_{x}, k_{y}, E_{b}\right) & =\sum_{i} a_{i}\left(E_{b}\right) \Phi_{i}\left(k_{x}, k_{y}\right) \\
& +b\left(E_{b}\right) I_{s u b}\left(k_{x}, k_{y}, E_{b}\right)+c\left(E_{b}\right)
\end{aligned}
$$

for a least square fitting of the measured APRES intensity 
$I\left(k_{x}, k_{y}, E_{b}\right)$. This function consists of a linear combination of all calculated orbital momentum maps $\Phi_{i}\left(k_{x}, k_{y}\right)$ that may contribute to the ARPES data, and a substrate term containing $I_{s u b}\left(k_{x}, k_{y}, E_{b}\right)$. The latter was obtained experimentally from a clean $\mathrm{Ag}$ (111) surface and included in the fitting in order to better reproduce sp-band features stemming from the substrate. An offset function $c\left(E_{b}\right)$ is also included. The parameters adjusted in the fit are $a_{i}\left(E_{b}\right), b\left(E_{b}\right)$ and $c\left(E_{b}\right)$, whereby fitting can be performed individually at each binding energy. The coefficients $a_{i}\left(E_{b}\right)$ represent the actual result. They can be understood as the (energy resolved) density of states projected onto a molecular orbital (projected density of states, PDOS) [8]. This is correct as long as the concerned orbitals are far enough from the Fermi level. If this condition is not fulfilled, as in the case of the (former) LUMO state, the fit result $a_{\mathrm{LUMO}}\left(E_{b}\right)$ must be divided by the Fermi distribution function in order to obtain the correct PDOS.

We would like to mention that we performed the fitting only on that part of the experimental data containing the intense molecular features. It has the shape of a ring covering the range $\left|\mathbf{k}_{||}\right|=1.25 \AA^{-1} \cdots 2.25 \AA^{-1}$ and is marked in Fig. 2c and d by two concentric dashed circles (black and white color is used for improving visibility). For smaller $\left|\mathbf{k}_{||}\right|$only very weak molecular features appear which are superimposed by strong substrate bands. Neglecting that part makes the fit much more stable and avoids artifacts stemming from the substrate bands without compromising the results.

In Fig. 3 we present the result of the orbital tomography analysis for the system under study. The PDOS $a_{\text {HOMO }}$ and $a_{\mathrm{LUMO}}$ for both molecules $\mathrm{A}$ and $\mathrm{B}$ are shown as a function of binding energy $E_{b}$, in the case of the LUMO after dividing by the Fermi distribution function for an effective temperature of $T_{\text {eff }}=930 \mathrm{~K}$. This is the reason for these curves exceeding the integrated ARPES intensity for the former LUMO in the vicinity of the Fermi energy. Note that $T_{\text {eff }}$ is not a real temperature. It accounts for temperature broadening of the Fermi distribution function $\left(T_{\text {sampe }}=300 \mathrm{~K}\right)$, and for a finite instrumental resolution of $150 \mathrm{meV}$. The integrated PES intensity for the entire circular disc is also shown in gray. The latter is what one would see in a conventional photoemission experiment, and shows no indications for an energy splitting of the orbitals of both molecules, neither for the HOMO nor for the LUMO. However, the result of the deconvolution algorithm reveals that molecule $\mathrm{B}$ has higher binding energies for both orbitals. While a relatively small difference of $40 \mathrm{meV}$ was found between the HOMO levels of both molecules, the difference between the LUMO peaks is much larger: $170 \mathrm{meV}$. These differences in the binding energies of the electronic states of molecules A and B stem from the complex interplay between intermolecular and molecule-substrate interactions.

Our results shall now be compared with scanning probe data. Kraft et al. [19] reported STS measurements that resolve both HOMO and LUMO resonances (they label

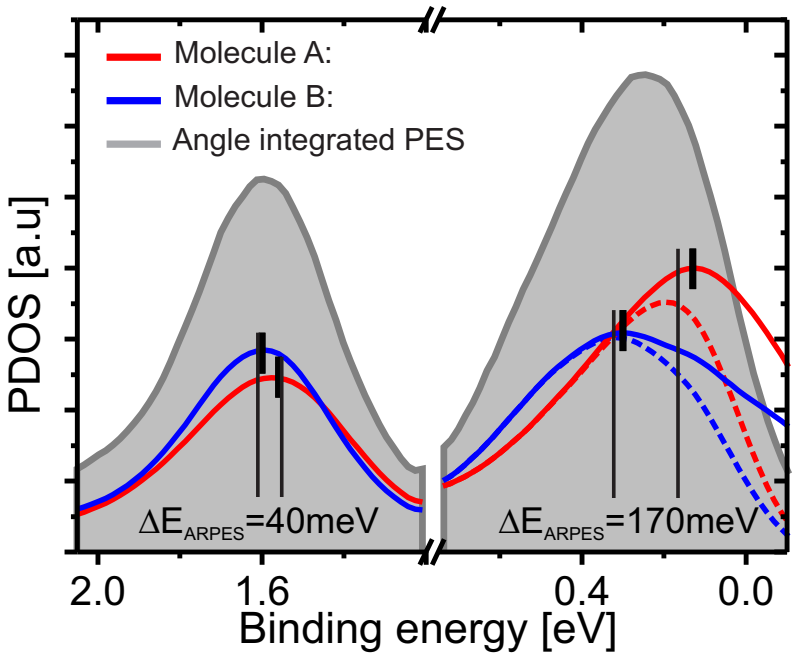

Fig. 3: Orbital projected density of states (PDOS) curves of the HOMO and LUMO level for both molecules in the PTCDA monolayer structure obtained by the orbital tomography analysis of the ARPES data. While the dotted curves for the LUMOs show the fitting parameters $a_{i}$, the solid curves are divided by the Fermi distribution function for an effective temperature of $930 \mathrm{~K}$. The gray-shadowed curve represents the ARPES data integrated over the ring in $k$-space which was used for fitting. Peak positions in the PDOS curves are indicated by vertical markers. Short thick and long thin lines mark the peak positions obtained from ARPES (this work) and STS (Ref. [19]), respectively

them "L1" and "L2", respectively) in their spectra for both molecules. They report very similar peak positions and shifts as found in the present study (peak positions are marked in Fig. 3 as vertical lines): The difference between molecule A and B for the LUMO resonance is $160 \mathrm{meV}$ (170 meV in our data), for the HOMO the values are even identical $(40 \mathrm{meV})$ within experimental errors. Even aspects of the lineshape are in agreement in the case of the LUMO states. Kraft et al. also mention the strong reduction of the HOMO-LUMO gap $(1.4 \mathrm{eV})$ compared to bulk PTCDA $(2.3 \mathrm{eV})$. Our data shows a HOMO-LUMO gap of $\approx 1.4 \mathrm{eV}$ for molecule $\mathrm{A}$ and $\approx 1.3 \mathrm{eV}$ for molecule $\mathrm{B}$. It can be put on record that the agreement between STS and ARPES results is excellent. Since both methods are known to be well comparable regarding energetic positions of molecular orbitals (in contrast to, e.g., transport gap measurements) [20] this agreement emphasizes the high reliability of the orbital tomography approach, also for adsorbate systems of high symmetry causing multi-domain interferences.

Precision of the tomographic deconvolution. So far the reliability of orbital tomography has not been discussed in broader detail. We do this in the following by testing two approaches, the first is based on a Monte Carlo simulation of the statistical uncertainty of experimental ARPES count rates, the second utilizes the linear regression algorithm which is used for the tomographic 
deconvolution procedure. The fitting function in equation (1) can be rewritten in the form

$$
F\left(k_{x}, k_{y}, E_{b}\right)=\sum_{i=1}^{p} \alpha_{i}\left(E_{B}\right) f_{i}\left(k_{x}, k_{y}\right)
$$

with (in our case) $p=4$ and fitting parameters $\alpha_{1}=$ $a_{A}\left(E_{B}\right), \alpha_{2}=a_{B}\left(E_{B}\right), \alpha_{3}=b\left(E_{B}\right)$ and $\alpha_{4}=c\left(E_{B}\right)$. The fitting functions $f_{i}$ are identified as $f_{1}=\Phi_{A}\left(k_{x}, k_{y}\right)$ and $f_{2}=\Phi_{B}\left(k_{x}, k_{y}\right)$, i.e., as the theoretical momentum maps calculated by the Fourier transforms of the orbitals under consideration of the molecular orientation, $f_{3}\left(k_{x}, k_{y}\right)$ as the measured substrate data $I_{s u b}\left(k_{x}, k_{y}\right)$, and $f_{4}$ as $f_{4}=1$ to take into account a possible $k$-independent background. The fitting is performed on a discrete, equidistant 2D-grid in $k$-space with $M$ grid-points:

$$
S=\sum_{k=1}^{M} w_{k}\left[I\left(k, E_{b}\right)-F\left(k, E_{b}\right)\right]^{2}
$$

Note that $k$ is here used as an index numbering all grid points $\left(k_{x}, k_{y}\right)$. The weights $w_{k}$ result from the Poisson-distributed counting statistics, thus $w_{k}$ is given by the inverse of the corresponding variance which in turn is proportional to the intensity at the $k$-th data point, $w_{k}=1 / I_{k} . \quad M$ usually describes the number of available data points used in the fitting algorithm. However, this is not true in our case. The experimental ARPES data are recorded for an equally spaced range of polar and azimuthal angles $(\phi, \theta)$ and then transformed onto an equidistant $\mathrm{k}$-space grid considering the threefold substrate symmetry. This results in a larger number of data points in the intensity cube $I\left(k_{x}, k_{y}, E_{b}\right)$ compared to the raw data. As discussed below this would artificially reduce the uncertainty of the orbital deconvolution. Hence, for a proper error analysis, the theoretical momentum maps $\Phi_{A}\left(k_{x}, k_{y}\right)$ and $\Phi_{B}\left(k_{x}, k_{y}\right)$ have to be transformed into the spherical coordinate system $(\phi, \theta)$ of the raw data. Alternatively the entire fitting procedure can be performed within the spherical coordinate system, which proved to give very similar results in our case.

We now focus on the our first approach for calculating the uncertainties of the fitting parameters $\alpha_{i}\left(E_{b}\right)$ using a Monte-Carlo based error analysis. A reference CBE data map is created from the fitting result, i.e., calculated $F\left(\phi, \theta, E_{b}\right)$ values fitting best to the experimental data. This reference momentum map represents the "ideal", noiseless intensity distribution of an ARPES experiment. The experimental noise is now simulated by adding a certain, arbitrary offset to each $(\phi, \theta)$ point of this reference CBE map. This offset is calculated individually for every point in the map using a random number generator according to a normal distribution with standard deviation $\sqrt{N}$, where $N$ is the count rate of that individual point. For high counting rates this corresponds very well to the expected Poisson distributed noise of the spectral data. The result is a reference $\mathrm{CBE}$ map with artificial noise which is now fitted according as described above, just as if it were experimental data. The resulting fit parameters $\alpha_{i}\left(E_{b}\right)$ will deviate from the original result since the reference map was modified by noise based on the experimental count rate. This procedure of calculating and fitting "noisy" data is now repeated many, e.g., 20 times, resulting in 20 different values for each fit parameter. Hence, the uncertainty of the fit parameters can simply be calculated as the statistical error for each of them, i.e., as the standard deviation of the fit parameter's distribution.

This Monte Carlo error analysis for the adsorbate system PTCDA $/ \operatorname{Ag}(111)$ results in error bars which are only in the range of $2 \%$ for all fitting parameters. This small error is due to the fact that the statistical noise does not strongly change the overall shape of the momentum map which has much more influence on the fitting result than the variation in the intensity of individual data points, as long as the variations are noise-like and not correlated or of systematic nature. The latter could be caused by the use of not ideal fitting pattern $f_{i}$, which can only be detected by the residual of the fitting procedure (which is also reflected in $S$ in equation (3)), but not in the uncertainties calculated by the Monte Carlo approach.

Such systematic errors are better treated by our second, alternative approach for calculating the errors in $\alpha_{i}\left(E_{b}\right)$. Here we make use of the fact that all fitting parameters enter equation (3) linearly. This allows us to solve the system of equations for each energy slice $E_{b}$ separately. This system of equations has the form

$$
\sum_{j} N_{i j} \alpha_{j}=v_{i}
$$

For a given energy slice, the matrix $N_{i j}$ and the vector $v_{j}$ are thus given by summing over all $M$ discrete $\{\phi, \theta\} \equiv \Omega$ points

$$
\begin{aligned}
N_{i j} & =\sum_{\Omega=1}^{M} w_{\Omega} f_{i}(\Omega) f_{j}(\Omega) \\
v_{i} & =\sum_{\Omega=1}^{M} w_{\Omega} f_{i}(\Omega) I\left(E_{b}, \Omega\right),
\end{aligned}
$$

By inverting Eq. (4), we obtain the desired orbitalprojected DOS, $a_{A}\left(E_{b}\right)$ and $a_{B}\left(E_{b}\right)$, as depicted in Fig. 3, as well as the substrate contribution $b\left(E_{b}\right)$ and the background $c\left(E_{b}\right)$. In addition to these results, we can also estimate their respective error bars by applying the standard expressions for uncertainty in the model parameters [21]. For the linear fit, the normal matrix $N_{i j}$ defined as the curvature matrix of $S$ with respect to the fit parameters $\alpha_{i}$,

$$
N_{i j}=\frac{1}{2} \frac{\partial^{2} S}{\partial \alpha_{i} \partial \alpha_{j}},
$$

is equal to Eq. (5). The standard deviations $\sigma$ and the correlations $\rho$ between the fit parameters are obtained from covariance matrix $C_{i j}$ given by the inverse of the normal 
matrix multiplied by the sum of least squares $S$ over the degrees of freedom $(M-p)$

$$
C_{i j}=\frac{S}{M-p}[N]_{i j}^{-1}
$$

Then, the standard deviations of the model parameters $\alpha_{i}$ are given by the square root of the diagonal terms

$$
\sigma_{\alpha_{i}}=\sqrt{C_{i i}}
$$

and the correlations between the fit parameters can be analyzed from

$$
r_{i j}=\frac{C_{i j}}{\sqrt{C_{i i} C_{j j}}}=\frac{[N]_{i j}^{-1}}{\sqrt{[N]_{i i}^{-1}[N]_{j j}^{-1}}}
$$

Values of $r_{i j}$ are in the range from -1 to +1 , where $r_{i j}=0$ indicates uncorrelated model parameters $\alpha_{i}$ and $\alpha_{j}$. The last conversion illustrates that the correlation between the fitting parameters only depends on the theoretical momentum maps used in the fitting procedure.

Performing this second approach of error analysis for the case discussed in this paper results in relative errors for all fitting parameters of also only $2 \%$, and therefore agrees very well with the Monte Carlo approach. The correlation factors are relatively high, $r_{A B}=-0.42$ for the LUMO and $r_{A B}=-0.68$ for the HOMO. However, the reason for rather small errors here is the very large number of grid points $M$ which appears in the denominator of eq. (8). Within the ring in $k$-space which we used for fitting more than $M=6000$ data points were measured.

Conclusion. - In order to verify the reliability of the ARPES orbital tomography for highly symmetric adsorbate systems we have chosen the monolayer of PTCDA/Ag(111). The experimental data was analyzed by deconvoluting the contributions of HOMO and (former) LUMO states of two symmetrically inequivalent molecules in the unit cell of the monolayer structure. The resulting projected densities of states were compared with scanning tunneling spectroscopy results reported earlier [19]. An excellent agreement could be achieved between both methods. Furthermore, we presented two types of error analyzes for the orbital tomography method. One simulates the experimental uncertainty caused by counting statistics in the ARPES measurement in a Monte Carlo approach, the second is based on the linear regression algorithm used for the tomographic deconvolution. Both approaches yield a relative uncertainty of the method of only $\approx 2 \%$. We can conclude that orbital tomography based on ARPES data, even though it represents an integrating method and hence has pretended disadvantages compared to a local probe technique like scanning tunneling spectroscopy, can provide reliable results even for complex surfaces with high symmetry and a correspondingly large number of equivalent domains.
We acknowledge financial support from the Deutsche Forschungsgemeinschaft (KU 1531/2-1), the Bundesministerium für Bildung und Forschung (GREKOS03SF0356B), and the Austrian Science Fond (FWF, P23190-N16). We also thank C. Schüssler-Langeheine, M. Ostler, T. Seyller and the staff of BESSY II / Helmholtz-Zentrum Berlin for their support during the beamtime.

\section{REFERENCES}

[1] Ueno N. and Kera S., Prog. Surf. Sci., 83 (2008) 490.

[2] Stadler C., Hansen S., Kröger I., Kumpf C., and Umbach E., Nature Phys., 5 (153) 2009.

[3] Kröger I., Stadtmüller B., Stadler C., Ziroff J., Kochler M., Stahl A., Pollinger F., Lee T.-L., Zegenhagen J., Reinert F., and Kumpf C., New J. Phys., 12 (083038) 2010.

[4] Stadtmüller B., Sueyoshi T., Kichin G., Kröger I., Soubatch S., Temirov R., Tautz F.S., and Kumpf C., Phys. Rev. Lett., 108 (106103) 2012.

[5] Stadtmüller B., Kröger I., Reinert F., and KumpF C., Phys. Rev. B, 83 (085416) 2011.

[6] Yoshida H. and Sato N., J. Phys. Chem. C, 116 (10033) 2012.

[7] Puschnig P., Berkebile S., Fleming A.J., Koller G., Emtsev K., Seyller T., Riley J.D., Ambrosch-Draxl C., Netzer F.P. and Ramsey M.G., Science, 326 (2009) 702.

[8] Puschnig P., Reinisch E.-M., Ules T., Koller G., Soubatch S., Ostler L., Romaner L., Tautz F.S., Ambrosch-Draxl C. and Ramsey M.G., Phys. Rev. B, 84 (2011) 235427.

[9] Wiessner M., Hauschild D., Schöll A., Feyer V., Winkler K. and Krömker B., Phys. Rev. B, 86 (2012) 045417.

[10] Berkebile S., Koller G., Fleming A.J., Puschnig P., Ambrosch-Draxl C., Emtsev K., Seyller T., RILey J.D. and Ramsey M.G., J. Electron Spectrosc. Relat. Phenom., 174 (2009) 22.

[11] Ziroff J., Forster F., Schöll A., Puschnig P. and Reinert F., Phys. Rev. Lett., 104 (2010) 233004.

[12] Duhm S., Gerlach A., Salzmann B., Bröker B., Johnson R., Schreiber F. and Koch N., Org. Electron., 9 (2008) 111.

[13] Zou Y., Kilian L., Schöll A., Schmidt T., Fink R. and Umbach E., Surf. Sci., 600 (2006) 1240.

[14] Tautz F.S., Prog. Surf. Sci., 82 (2007) 479.

[15] Schwalb C.H., Sachs S., Marks M., Schöll A., Reinert F., Umbach E., and Höfer U., Phys. Rev. Lett., 101 (146801) 2008.

[16] Glöckler K., Seidel C., Soukopp A., Sokolowski M., UMbach E., BÖhringer M., Berndt R., and Schneider W.-D., Surf. Sci., 405 (1) 1998.

[17] Hauschild A., Temirov R., Soubatch S., Bauer O., SchÖll A., Cowie B.C.C., LeE T.-L., Tautz F.S. and Sokolowski M., Phys. Rev. B, 81 (2011) 125432. 
[18] Kröger I., Stadtmüller B., Wagner C., Weiss C., Temirov R., Tautz F.S. and Kumpf C., J. Chem. Phys., 135 (2011) 234703.

[19] Kraft A., Temirov R., Henze S.K.M., Soubatch S., Rohlfing M. and Tautz F.S., Phys. Rev. B, 74 (2006) 041402.

[20] Kilian L., Hauschild A., Temirov R., Soubatch S., Schöll A.,Bendounan A., Reinert F., Lee T.-L., Tautz F.S., Sokolowski M. and Umbach E., Phys. Rev. Lett., 100 (2008) 136103.

[21] Wolberg J., Data Analysis Using the Method of Least Squares: Extracting the Most Information from Experiments (Springer) 2006. 